Ophthalmologe 2007 · 104:1066-1067

DOI 10.1007/s00347-007-1503-3

Online publiziert: 20. April 2007

๑) Springer Medizin Verlag 2007
J.C. Paarlberg ${ }^{1}$. J.C. den Hollander ${ }^{2}$. F. Hafezi ${ }^{3}$. D. Paridaens ${ }^{1}$

${ }^{1}$ Department of Oculoplastic \& Orbital Surgery, The Rotterdam Eye Hospital

2 Department of Pathology, Erasmus Medical Centre, Rotterdam

${ }^{3}$ IROC, Institute for Refractive and Ophthalmic Surgery, Zürich

\title{
Adenoid-zystisches Karzinom des Oberlids
}

\section{Anamnese}

Ein 57-jähriger Patient wurde uns mit einer seit 2 Jahren schmerzlos wachsenden Läsion des rechten Oberlids überwiesen. Diese wurde zuvor als „Chalazion“ diagnostiziert und mit topischen Antibiotika sowie wiederholten Exzisionen behandelt, ohne dass eine histopathologische $\mathrm{Ge}$ websuntersuchung durchgeführt wurde.

\section{Klinischer Befund}

Die klinische Untersuchung zeigte einen $2,0 \times 2,5 \mathrm{~cm}$ großen, multinodulären Tumor, welcher die medialen zwei Drittel des Oberlids einnahm (• Abb. 1). Dieses wies eine Blepharoptose von $3 \mathrm{~mm}$ auf. Die regionalen Lymphknoten waren palpatorisch unauffällig. Der Tumor wurde unter Wahrung des Lidrands und eines Sicherheitsabstands totalexzidiert. Das $3,5 \times 3,0 \times 1,2 \mathrm{~cm}$ große resezierte $\mathrm{Ge}$ webestück mit einem Durchmesser von knapp 2,o cm wies einen weißlichen, soliden, nichtzystischen multinodulären Aspekt auf.

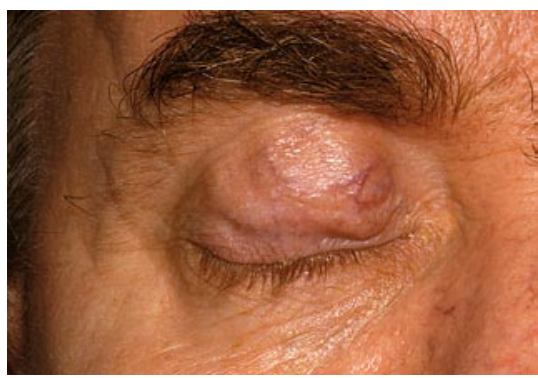

Abb. $1 \Delta$ Ausgedehntes adenoid-zystisches Karzinom des Oberlids

\section{Diagnostik}

Die mikroskopische Untersuchung ergab einen subkonjunktivalen epithelialen Tumor mit cribriformem Wachtumsmuster sowie mikrozystische Räume, welche mit mukoidem Material angefüllt waren (- Abb. 2a). Die Zellen enthielten runde bis ovale hyperchromatische Nuklei und nur wenig Zytoplasma, was die klinische Diagnose eines adenoid-zystischen Karzinoms bestätigte. Fokal wude zudem eine Insel von bereits vorhandenem akzessorischem Tränendrüsengewebe bestimmt. Die Schnittränder waren frei von Tumor, ein perineurales Wachstum konnte nicht festgestellt werden.

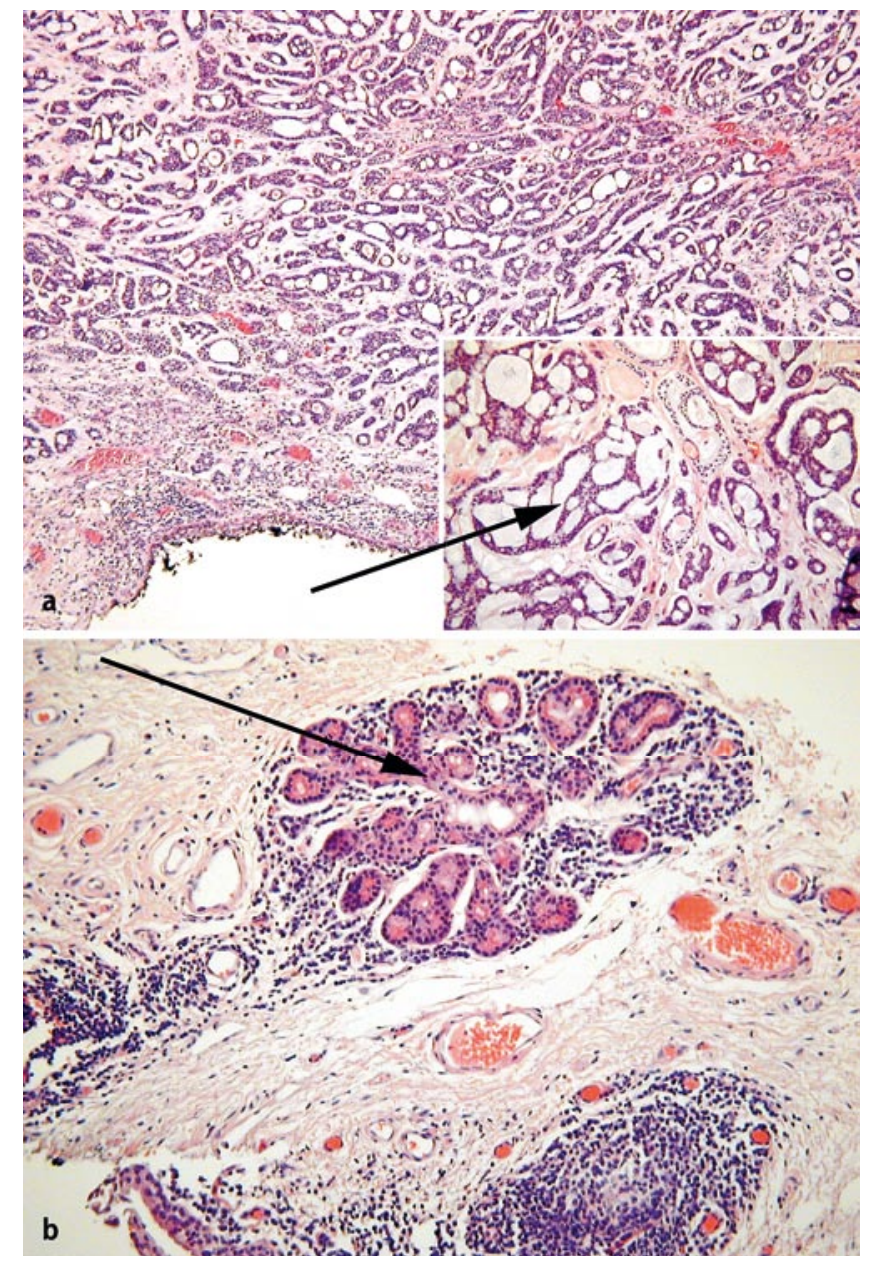

Abb. $2<$ a Histologisch zeigte sich unter der Konjunktiva ein adenoid-zystischen Karzinom mit cribriformem Muster (Pfeil). Die zystischen Räume sind mit mukoidem Material ausgefüllt (HE-Färbung, Vergr. 40:1; Inset: HEFärbung, Vergr. 200:1). b Bereits vorhandenes akzessorisches Tränendrüsengewebe (Pfeil) in der tarsalen Bindehaut (HE-Färbung, Vergr. 200:1) 


\section{Therapie und Verlauf}

Das Oberlid wurde nach der primären Exzision rekonstruiert. Der postoperative Verlauf war unauffällig und $1 \mathrm{Jahr}$ nach der Behandlung zeigte sich ein gutes kosmetisches und funktionelles Resultat ohne Rezidive.

\section{Diskussion}

Das adenoid-zystische Karzinom kann in jedem Lebensalter auftreten, zeigt aber eine Häufung in der 5. Lebensdekade [6]. Periokulär findet sich das adenoid-zystische Karzinom am häufigsten in der Tränendrüse [6]. Andere Lokalisationen umfassen die Schweißdrüsen [7, 8], ektopisches [10] und akzessorisches [3] Tränendrüsengewebe sowie den Tränensack [9].

Während adenoid-zystische Karzinome der Tränendrüse typischerweise rasch zu Symptomen wie Schmerz und Gefühllosigkeit führen [6], sind Patienten bei Befall der akzessorischen Tränendrüsen oder anderen periokulären Geweben häufig symptomlos $[7,8,10]$. Dies kann wie in diesem Fall dazu führen, dass der Arztbesuch erst in einem fortgeschrittenen Stadium erfolgt. Bösartige Tumoren der akzessorischen Tränendrüsen wurden bisher nur selten beschrieben, eine Literatursuche ergab ein mukoepidermoides [2], ein unspezifisches [4] und ein adenoid-zystisches Karzinom des superonasalen konjunktivalen Fornix [3] sowie des Unterlids [5]. Im vorliegenden Fall lag eine reine Oberlidschwellung ohne Beteiligung der Lidkante vor. Differenzialdiagnostisch sollte auch ein primär kutanes adenoidzystisches Karzinom berücksichtigt werden. In der Frühphase kann ein solcher Tumor durchaus den Aspekt eines Chalazions aufweisen, jedoch sollte die Tatsache der „leeren Kürette“ [1] den Chirurgen dazu veranlassen, eine Gewebebiopsie durchzuführen.

Dieser Fall soll vor Augen führen, dass das adenoid-zystische Karzinom in die Differenzialdiagnose der langsam wachsenden Lidtumoren miteinbezogen werden sollte.
Korrespondenzadresse

\section{Dr. Dr. D. Paridaens}

Department of Oculoplastic \& Orbital Surgery,

The Rotterdam Eye Hospital

Schiedamsevest 180, 3011 Rotterdam

Niederlande

paridaens@icapi.nl

Danksagung. Wir danken der SWOO-Stiftung für die finanzielle Unterstützung bei dieser Arbeit.

Interessenkonflikt. Es besteht kein Interessenkonflikt. Der korrespondierende Autor versichert, dass keine Verbindungen mit einer Firma, deren Produkt in dem Artikel genannt ist, oder einer Firma, die ein Konkurrenzprodukt vertreibt, bestehen. Die Präsentation des Themas ist unabhängig und die Darstellung der Inhalte produktneutral.

\section{Literatur}

1. Cunniffe G, Chang BY, Kennedy S et al. (2002) Beware the empty curette! Orbit 21: 177-180

2. Dithmar S, Wojno TH, Washington C et al. (2000) Mucoepidermoid carcinoma of an accessory lacrimal gland with orbital invasion. Ophthal Plast Reconstr Surg 16: 162-166

3. Duke TG, Fahy GT, Brown LJ (2000) Adenoid cystic carcinoma of the superonasal conjunctival fornix. Orbit 19: 31-35

4. Freydinger JE, Duhig JT (1964) Carcinoma of accessory lacrimal gland. Arch Pathol 77: 643-645

5. Goto H, Yamamoto T, Ishiyama Z et al. (2006) Adenoid cystic carcinoma arising from the lower eyelid. Jpn J Ophthalmol 50: 374-376

6. Jakobiec FA, Bilyk JR, Font RL (1996) Orbit, in Ophthalmic pathology: an atlas and textbook. In: Spencer WH (ed) WB Saunders, Philadelphia, pp 2494-2509

7. Marback EF, Costa AL, Nossa LM et al. (2003) Eyelid skin adenoid cystic carcinoma: a clinicopathological study of one case simulating sebaceous gland carcinoma. Br J Ophthalmol 87: 118-119

8. Mencia-Gutierrez E, Gutierrez-Diaz E, Ricoy JR et al. (2001) Primary cutaneous adenoid cystic carcinoma of the eyelid. Am J Ophthalmol 131: 281-283

9. Parmar DN, Rose GE (2003) Management of lacrimal sac tumours. Eye 17: 599-606

10. Shields JA, Shields CL, Eagle RC Jr et al. (1997) Adenoid cystic carcinoma developing in the nasal orbit. Am J Ophthalmol 123: 398-399
Ophthalmologe 2007 · 104:1066-1067

DOI 10.1007/s00347-007-1503-3

๑) Springer Medizin Verlag 2007

J.C. Paarlberg · J.C. den Hollander .

F. Hafezi $\cdot$ D. Paridaens

Adenoid-zystisches

Karzinom des Oberlids

Zusammenfassung

Das adenoid-zystische Karzinom ist ein bösartiger epithelialer Tumor mit langsamem Wachstum, welcher vor allem die Tränendrüse, sehr selten auch akzessorisches Tränendrüsengewebe befallen kann. Durch sein perineurales Vordringen sind lokale Rezidive nach Exzision ein häufiges therapeutisches Problem. In diesem Beitrag stellen wir einen Patienten mit einem ausgedehnten adenoid-zystischen Karzinom des Oberlids vor, welches fehldiagnostiziert und als Chalazion (ohne histologische Bestätigung) behandelt wurde. Seinen Ursprung nahm dieser Tumor aller Wahrscheinlichkeit nach aus akzessorischem Tränendrüsengewebe.

Schlüsselwörter

Adenoid-zystisches Karzinom · Lidtumoren . Chalazion

\section{Adenoid cystic carcinoma of the upper eyelid}

\section{Abstract}

Adenoid cystic carcinoma (ACC) is a rare epithelial malignancy, which tends to grow slowly. ACC is an intractable neoplasm due to its ability to invade perineural spaces. Local recurrence after excision is not unusual. ACC most commonly arises in the lacrimal gland. Very rarely, ACC originates from accessory lacrimal gland tissue. Here, we present a patient with a large ACC of the central upper eyelid, which had been misdiagnosed and treated as a chalazion without histological examination. Its origin most likely is an accessory lacrimal gland.

\section{Keywords}

Adenoid cystic carcinoma - Eyelid neoplasms . Chalazion 